\title{
Neuropsychiatric Symptoms and Global Functional Impairment along the Alzheimer's Continuum
}

\author{
Lauren P. Wadsworth ${ }^{\mathrm{b}}$ Natacha Lorius ${ }^{\mathrm{a}, \mathrm{b}}$ Nancy J. Donovan ${ }^{\mathrm{a}, \mathrm{d}}$ \\ Joseph J. Locascio ${ }^{b}$ Dorene M. Rentz ${ }^{a, b}$ Keith A. Johnson ${ }^{a, c}$ \\ Reisa A. Sperling ${ }^{a, b}$ Gad A. Marshall ${ }^{a, b}$ for the Alzheimer's Disease \\ Neuroimaging Initiative
}

${ }^{a}$ Center for Alzheimer Research and Treatment, Department of Neurology, Brigham and Women's Hospital,

Harvard Medical School, Departments of beurology and 'Radiology, Massachusetts General Hospital, Harvard

Medical School, Boston, Mass., and dDepartment of Psychiatry, Cambridge Health Alliance, Cambridge, Mass., USA

\section{Key Words}

Alzheimer's disease $\cdot$ Anxiety $\cdot$ Apathy $\cdot$ Disease progression $\cdot$ Hallucinations $\cdot$ Mild cognitive impairment $•$ Neuropsychiatric symptoms

\section{Abstract}

Background/Aims: Neuropsychiatric symptoms in Alzheimer's disease (AD) are highly prevalent. We sought to determine whether neuropsychiatric symptoms were related to global functional impairment at baseline and over a 3-year period in older normal control (NC), mild cognitive impairment (MCI) and mild AD dementia subjects. Methods: Eight hundred and twelve subjects (229 NC, $395 \mathrm{MCl}, 188$ AD) from the Alzheimer's Disease Neuroimaging Initiative study underwent cognitive and behavioral assessments over 3 years. Results: Greater hallucinations, anxiety and apathy were associated with greater global functional impairment at baseline, while the presence of hallucinations and apathy at baseline was associated with greater global functional impairment over time across all subjects. The following neuropsychiatric symptoms were not significantly associated with global functioning: delusions, agitation, depression, euphoria, disinhibition, irritability, aberrant motor behaviors, sleep and appetite. Conclusions: These results suggest that increased baseline hallucinations, apathy and anxiety are associated with current and future disease progression in AD.

Copyright $\odot 2012$ S. Karger AG, Basel

\section{Introduction}

Neuropsychiatric symptoms in the Alzheimer's disease (AD) spectrum are multidimensional and highly prevalent across the continuum of amnestic mild cognitive impairment (MCI) and $\mathrm{AD}$ dementia. These symptoms are also of interest as potential manifestations of underlying disease at the earliest stages of $\mathrm{AD}$ prior to a diagnosis

Data used in the preparation of this article were obtained from the Alzheimer's Disease Neuroimaging Initiative (ADNI) database (www.loni.ucla.edu\ADNI). The authors are site investigators and research staff for the ADNI at Brigham and Women's Hospital and Massachusetts General Hospital. The other site investigators within the ADNI contributed to the design and implementation of the ADNI and/or provided data but did not participate in the analysis or writing of this report. A complete list of ADNI investigators is available at www.loni.ucla.edu $\backslash A D N I \backslash$ Collaboration $\backslash A D N I$ Authorship_list.pdf.

\section{KARGER}

Fax +41613061234

E-Mail karger@karger.ch

www.karger.com
(C) 2012 S. Karger AG, Basel

$1420-8008 / 12 / 0342-0096 \$ 38.00 / 0$

Accessible online at:

www.karger.com/dem
Gad A. Marshall, MD

Center for Alzheimer Research and Treatment

Brigham and Women's Hospital

221 Longwood Avenue, BL-104H

Boston, MA 02115 (USA)

Tel. +1 617732 8085, E-Mail gamarshall@partners.org 
of MCI or dementia [1]. In population-based studies of MCI, elevated rates of depression (20\%), apathy (15\%), irritability (15\%), agitation (11\%), sleep disturbance (14\%), anxiety (10\%), hallucinations (1.3\%) and other behavioral symptoms occur at intermediate frequencies between cognitively normal elderly and dementia patients [1]. The total neuropsychiatric burden typically increases over the course of $\mathrm{AD}$; however, while symptoms such as apathy endure and are more prevalent in severe dementia, other symptoms such as irritability and anxiety may occur episodically over time [1-3]. The role of neuropsychiatric symptoms at the very earliest stages of $\mathrm{AD}$, prior to significant cognitive impairment, is less well defined.

Neuropsychiatric rather than cognitive symptoms accompanying $\mathrm{AD}$ dementia have been shown to be the primary determinants of caregiver distress and the need for formal and informal care; furthermore, neuropsychiatric symptoms have cumulative effects on caregiver burden with increasing numbers of symptoms $[4,5]$. These symptoms are associated with reduced quality of life for the caregiver and patient and heavily influence costs of care and the risk of institutionalization [6-10]. Although critical features of $\mathrm{AD}$, neuropsychiatric symptoms are heterogeneous and have not been fully characterized with respect to their individual contribution to $\mathrm{AD}$-related impairments and outcomes, particularly in the earlier stages of the disease spectrum. Only recently has it been suggested to formally integrate them into disease staging criteria along with cognitive and functional symptoms; in the new $\mathrm{AD}$ dementia criteria, behavioral changes can count toward the minimum of two clinical deficits, which previously included only cognitive changes [11].

Particular attention has been directed at depression and apathy as early manifestations of $\mathrm{AD}$ symptomatology and as possible predictors of progression from MCI to dementia [12-14]. Apathy is the most common behavioral symptom in $\mathrm{AD}$, occurring in $55 \%$ of dementia patients in the European Alzheimer's Disease Consortium study and present in 11-53\% of MCI patients as shown by a recent systematic review [15]. Apathy has been associated with executive dysfunction in $\mathrm{AD}$ dementia $[16$, 17]. Apathy has also been associated with impairment in activities of daily living (ADL), which in turn has been associated with executive dysfunction in $\mathrm{MCI}$ and $\mathrm{AD}$ dementia $[18,19]$. Other neuropsychiatric symptoms such as anxiety, agitation and delusions/hallucinations have also been examined as predictors of functional decline and markers of disease progression [3, 13, 20-24].

The objective of this study was to investigate the relationship between individual neuropsychiatric symptoms and global functional impairment both cross-sectionally and longitudinally across a spectrum of older normal control (NC), MCI and mild AD dementia subjects in a large, well-defined population. We included the NC group because it represents an at-risk group that might be in the preclinical stage of $\mathrm{AD}$. We then examined the relationship between neuropsychiatric symptoms and progression from NC to MCI and from MCI to AD dementia. We focused on individual neuropsychiatric symptoms rather than clusters of symptoms because at these early stages of $\mathrm{AD}$, there are fewer neuropsychiatric symptoms and they might not cluster as clearly as they do later on in the disease. The analyses performed here accounted for various factors which have not always been controlled for in other studies. Those included diagnostic group, sex, age, duration of $\mathrm{AD}$ symptoms, apolipoprotein E $\varepsilon 4$ (APOE4) carrier status, cognitive reserve, memory performance, processing speed and use of antidepressant medications. We hypothesized that greater individual neuropsychiatric symptoms at baseline, similarly to greater cognitive impairment at baseline, will be associated with proxies of disease severity and progression, including greater global functional impairment at baseline and over time and progression from a milder to a more impaired diagnosis.

\section{Materials and Methods}

\section{Subjects}

The data used in the preparation of this article were obtained from the Alzheimer's Disease Neuroimaging Initiative (ADNI) database (www.loni.ucla.edu\ADNI) [25]. ADNI is a multicenter, natural history trial consisting of $\mathrm{NC}$, amnestic MCI and mild AD dementia subjects followed with longitudinal periodic imaging of multiple modalities, blood and cerebrospinal fluid analyses and clinical and neuropsychological assessments. The primary goals of the ADNI include using these various assessments to measure the progression of MCI and mild AD dementia, determining the sensitivity and specificity of biomarkers in serving as surrogate outcome measures in treatment trials and reducing the time and cost of clinical trials. The ADNI is the result of efforts of many coinvestigators from a broad range of academic institutions and private corporations, and subjects have been recruited from over 50 sites across the USA and Canada.

The study population was thoroughly described in a previous report [19]. Eight hundred and twelve subjects from the ADNI study (diagnoses at baseline: $229 \mathrm{NC}, 395 \mathrm{MCI}, 188$ $\mathrm{AD}$ dementia) underwent cognitive testing and behavioral assessments at baseline and up to 5 more times over a period of 3 years. At screening, subjects were aged 55-91 years (inclusive; NC subjects were aged 60-90 years), medically stable and had study partners able to provide collateral information. Also, subjects did not have significant neurological conditions 
(other than $\mathrm{MCI}$ or $\mathrm{AD}$ dementia), recent alcohol or substance abuse or active psychiatric diagnoses (such as major depressive disorder or bipolar disorder) at screening, as determined by the site investigator. Subjects were not significantly depressed (Geriatric Depression Scale [26] short form $\leq 5$ ) and did not have significant cerebrovascular risk factors (Modified Hachinski Ischemic Score [27] $\leq 4)$.

The diagnostic group (NC, MCI or AD dementia) was determined by site investigators at screening and baseline visits. For this study, we used the diagnosis from baseline since that time point included extensive neuropsychiatric and neuropsychological data. As per the ADNI protocol, quantitative data, as well as more qualitative global assessments, were used by site investigators to assign subjects to diagnostic groups. Final diagnoses were based on the site investigator's clinical judgment. The diagnostic group criteria are detailed below.

At screening, NC subjects had a Clinical Dementia Rating (CDR) [28] global and memory box score of 0 and a MiniMental State Examination (MMSE) [29] score of 25-30 (inclusive). NC subjects had no significant impairment in individual cognitive domains. Moreover, they performed within 1.5 standard deviations of education-adjusted cutoff scores on the Logical Memory IIa (LM-IIa; delayed recall) of the Wechsler Memory Scale-Revised [30] (subjects with $\geq 16$ years of education required an LM-Ila score $>8$; subjects with 8-15 years required LM-IIa $>4$; subjects with $0-7$ years required LM-IIa $>2$ ).

MCI subjects met the following criteria for single- or multiple-domain amnestic MCI [31]: memory complaint by subject or study partner; objective memory impairment (1.5 standard deviations below education-adjusted cutoff scores on the LM-IIa of the Wechsler Memory Scale-Revised); essentially preserved instrumental ADL (this determination was based on a qualitative clinical assessment by each site investigator; a specific cutoff score on a test of ADL was not used to determine this), and not demented. At screening, MCI subjects had a global CDR score of 0.5 , a CDR memory box score $\geq 0.5$ and MMSE score of 24-30 (inclusive).

$\mathrm{AD}$ dementia subjects met the National Institute of Neurologic and Communicative Disorders and Stroke and the $\mathrm{AD}$ and Related Disorders Association Work Group criteria for probable AD [32] with mild dementia severity. At screening, they had a global CDR score of 0.5 or 1, an MMSE of 20-26 (inclusive) and the same objective memory impairment scores as MCI subjects.

The ADNI study was approved by the local institutional review board of each participating site. Prior to performance of any study procedures, informed consent was obtained from all subjects and study partners.

\section{Clinical Assessments}

The Neuropsychiatric Inventory brief questionnaire form (NPI-Q) [33] was used to assess neuropsychiatric symptoms. The NPI-Q is an informant-based, well-validated questionnaire, used widely in the research setting, which consists of the following 12 items: delusions, hallucinations, agitation/aggression, depression/dysphoria, euphoria/elation, anxiety, apathy, disinhibition, irritability/lability, aberrant motor behaviors, sleep and appetite/eating disorder. The NPI-Q includes one question for each of the 12 items, which is answered either yes or no (indicating the presence or absence of the symptom) and if present rated for severity. Severity scores for each NPI-Q item were used as predictors of interest in the cross-sectional analysis of this study (higher scores indicate greater severity of the neuropsychiatric symptom; range $0-3$ ). We did not have access to more specialized and in-depth assessments of each neuropsychiatric symptom, which might have made it possible to have a richer construct for each relevant symptom.

As indicated above, in our primary analyses we utilized the individual NPI-Q items because we wanted to determine if at the early stages of $\mathrm{AD}$, particular neuropsychiatric symptoms rather than clusters of symptoms would be associated with proxies of disease severity and progression. We also performed a factor analysis using all 12 NPI-Q items, which yielded 2 factors (clusters), namely an Affective factor (consisting of disinhibition, apathy, irritability, agitation, appetite, euphoria, anxiety and depression) and a Psychotic factor (consisting of hallucinations, delusions, sleep and aberrant motor behaviors). The Affective factor was significantly correlated with apathy severity $(\mathrm{r}=0.64, \mathrm{p}<0.0001)$ and anxiety severity $(\mathrm{r}=0.51, \mathrm{p}<$ 0.0001 ), while the Psychotic factor was significantly correlated with hallucination severity $(\mathrm{r}=0.70, \mathrm{p}<0.0001)$ and anxiety severity $(r=0.47, p<0.0001)$. The Affective factor was also significantly correlated with the Psychotic factor $(\mathrm{r}=0.26, \mathrm{p}<$ 0.0001 ). These factors were used in subsequent analyses.

The CDR [28] was used to assess global functional impairment at baseline and global functional decline over time. The $\mathrm{CDR}$ is an informant- and subject-based scale which is well validated and widely used in research and clinical practice. The CDR assesses the following 6 domains: memory, orientation, judgment and problem solving, community affairs, home and hobbies, and personal care. A global score of $0-3$ and sum of boxes (SB) score of $0-18$ are generated from the scale. The CDR-SB was used as the dependent variable in most of the analyses in this study (higher scores indicate greater impairment; range 0-18).

The following cognitive scales were also used for this study, either for inclusion criteria as noted above or as predictors serving as covariates in the various analyses. The MMSE was used to assess global cognitive function (range 0-30; lower scores on the MMSE indicate greater cognitive impairment). The Rey Auditory Verbal Learning Test (RAVLT) [34] assesses episodic memory performance (the Total Learning score for words recalled over 5 learning trials was used in this analysis; range $0-75$; lower scores on the RAVLT indicate greater memory impairment). The Wechsler Adult Intelligence Scale-Revised Digit Symbol subtest [35] assesses processing speed, visual scanning and complex attention (possible range $0-110$; in the current analyses, the highest score was 80; lower scores on Digit Symbol indicate greater impairment). In a previous analysis of the ADNI database, we found that the Digit Symbol subtest was a more robust measure than the more traditional executive function measure of the Trail Making Test B, which had a narrower range of scores and was not as normally distributed [19]. The American National Adult Reading Test (AMNART) intelligence quotient (IQ) [36] provides an estimate of premorbid 
verbal intelligence and serves as a proxy of cognitive reserve (an error score was converted into an IQ score; IQ score range 74-132; higher AMNART IQ scores indicate a higher level of premorbid intelligence). We used AMNART IQ rather than education in our analyses because in this sample, as in many others, males had more years of education than females $(t=5.2$, $\mathrm{p}<0.0001)$, while both had the same AMNART IQ score $(\mathrm{t}=$ $-0.9, \mathrm{p}=0.39$ ). Therefore, by using AMNART IQ we avoided the confounding association with sex.

APOE4 carrier status was determined and reported as one of the following three designations: noncarrier, heterozygous carrier or homozygous carrier. Psychotropic medication use (dichotomous, i.e. present/absent) at baseline was also assessed, including the following five medication categories: antidepressants (present for $20.7 \%$ of subjects), anxiolytics (1.4\%), mood stabilizers $(0.1 \%)$, antipsychotics $(0.4 \%)$ and sleeping agents (1.2\%). Only antidepressant use was included as a predictor in the analyses because the rest of the medications were used at extremely low frequencies. Duration of $\mathrm{AD}$ symptoms (in years) was available only for subjects in the mild AD dementia diagnostic group at study entry and was included as a predictor. It was set to 0 for $\mathrm{NC}$ and MCI subjects in order to include them in analyses involving duration as a predictor/covariate, and therefore it is interpreted as an estimate of only the duration of illness subsequently clinically diagnosed as AD dementia.

\section{Statistical Analyses}

All analyses in this study were carried out using SAS Version 9.2 and SPSS Version 20. Associations among diagnostic groups, demographic variables and covariates were evaluated using the $\chi^{2}$ test for categorical variables and analysis of variance with Bonferroni correction for continuous variables.

\section{Cross-Sectional Analysis}

A general linear regression model approach was employed for our cross-sectional analysis (using the SAS GLM and GLMSelect procedures) with backward elimination of predictors using a $\mathrm{p}<0.01$ retention requirement (a cutoff of 0.05 was considered too liberal given that a series of significance tests would be applied before arriving at the final retained model). Residuals from the final model were examined graphically to ensure that their distributions reasonably satisfied model assumptions of normality and homoscedasticity. Given the large sample sizes in this study, it was possible for effects of small substantive or clinical importance to be statistically significant. Therefore, reported significance test results were complemented with effect size estimates such as partial regression coefficient estimates $(\mathrm{t}$ ) and confidence intervals (CIs) thereof, estimates of percent variance accounted for in the dependent variable by the model as a whole and the portion of this variance uniquely accounted for by each predictor term individually (adjusting for the other predictors).

The dependent variable for this model was CDR-SB. The predictors included the 12 NPI-Q items and the interaction of each item with diagnosis, diagnosis and sex main effects and their interaction, age (linear and quadratic effects), duration of AD symptoms, APOE4 carrier status, AMNART IQ, RAVLT Total Learning score, Digit Symbol score and antidepressant use. The inclusion of the interaction of NPI-Q items with diagnosis allowed us to test for any differential relation of any given NPI-Q item to CDR-SB across diagnostic groups. Should the interaction be significant, a test of the same relation could be followed up separately within each group, with these multiple $\mathrm{p}$ values 'protected' by the required significant preliminary omnibus interaction. On the other hand, if the interaction is eliminated as nonsignificant, a then presumably homogeneous within-group relation (or lack of same) of NPI-Q item to CDR-SB would be tested for significance, pooling strength and power from across all groups.

\section{Longitudinal Analyses: Mixed Effects Model}

A mixed random and fixed coefficient regression model was employed (using the SAS Mixed procedure) for our longitudinal analyses in order to ascertain the effects of the predictors on the trajectory of change across time in the dependent variable, as previously described [37]. A backward elimination procedure (using a cutoff of $\mathrm{p}=0.05$ ) was used on a large initial pool of fixed predictors and variances/covariances of random terms. In this analysis, time in the study (in years) was the only predictor modeled as both random and fixed. An intercept and linear slope term for time was analyzed with no nonlinear terms because the relatively few observations across time per subject did not readily permit polynomial or other nonlinear terms. The random intercept and slope were allowed to freely correlate. With respect to fixed predictors, the baseline score for the dependent variable was removed from the dependent variable side of the prediction equation, and both it and its interaction with time were included as predictors in order to adjust for differences in level and trajectory over time of the dependent variable due to different baseline starting points. Residuals with respect to fixed and both random as well as fixed predictors from the final retained model were examined graphically to ensure that assumptions of normality and homoscedasticity of their distribution were reasonably satisfied. The squared correlations of predicted values from fixed and random predictor sets versus actual values were also used to indicate the percent of variance of the dependent variable linearly accounted for by the predictors.

Longitudinal CDR-SB was the dependent variable. The fixed predictors included baseline NPI-Q items (informed by the results of the cross-sectional analysis; items included were hallucinations, anxiety and apathy) and their interaction with time, as well as the covariates used in the cross-sectional analysis, and the baseline dependent variable and its interaction with time. The random predictors included correlated intercepts and linear slopes of time.

This analysis was repeated with the Affective factor and Psychotic factor instead of the individual NPI-Q items.

Longitudinal Analyses: Cox Proportional Hazards Model

A Cox proportional hazards model was employed to test for prediction of time to change in diagnosis from a baseline of MCI to an endpoint of $\mathrm{AD}$ dementia. We employed a separate analogous analysis where the baseline diagnosis was $\mathrm{NC}$ and the endpoint was MCI. Only data for subjects whose earliest diagnosis was the baseline diagnosis of interest were included in a 
Table 1. Baseline demographic and clinical data for our subjects

\begin{tabular}{lccc}
\hline Group & NC & MCI & AD dementia \\
\hline Number & 229 & 395 & 188 \\
Age, years & $76.0 \pm 5.0(60-90)$ & $74.8 \pm 7.5(55-90)$ & $75.3 \pm 7.5(55-91)$ \\
Male, \% & $52.0^{\mathrm{e}}$ & 64.3 & 51.6 \\
Education, years & $16.0 \pm 2.9(6-20)^{\mathrm{d}}$ & $15.7 \pm 3.1(4-20)$ & $14.7 \pm 3.1(4-20)$ \\
AMNART IQ (possible range 74-132) & $121.1 \pm 10.6(75-132)^{\mathrm{c}}$ & $116.6 \pm 11.5(74-132)$ & $114.0 \pm 11.7(77-132)$ \\
AD symptom duration, years & & & $3.5 \pm 2.5(0-13)$ \\
APOE4 noncarrier/heterozygous carrier/ & & & \\
$\quad$ & $72.9 / 24.5 / 2.2^{\mathrm{b}}$ & $46.6 / 41.8 / 11.4$ & $34.0 / 46.8 / 19.1$ \\
homozygous carrier, \% & $29.1 \pm 1.0(25-30)^{\mathrm{a}}$ & $27.04 \pm 1.8(24-30)$ & $23.3 \pm 2.0(20-26)$ \\
RAVLT Total Learning (possible range 0-75) & $43.1 \pm 10.0(5-69)^{\mathrm{a}}$ & $30.8 \pm 9.0(11-68)$ & $23.19 \pm 7.6(5-42)$ \\
Digit Symbol (possible range 0-110) & $45.8 \pm 10.2(18-80)^{\mathrm{a}}$ & $36.8 \pm 11.3(1-69)$ & $26.5 \pm 13.2(0-62)$ \\
CDR-SB (possible range 0-18) & $0.0 \pm 0.1(0-0.5)^{\mathrm{a}}$ & $1.6 \pm 0.9(0.5-5)$ & $4.3 \pm 1.6(1-9)$ \\
Antidepressant use, \% & $10.0^{\mathrm{b}}$ & 21.5 & 31.9
\end{tabular}

All values represent means \pm standard deviation (range), except where indicated otherwise. ${ }^{a} \mathrm{p}<0.0001$ for $\mathrm{NC}$ vs. MCI, NC vs. AD and MCI vs. AD; ${ }^{\mathrm{b}} \mathrm{p}<0.01$ for $\mathrm{NC}$ vs. MCI, NC vs. $\mathrm{AD}$ and $\mathrm{MCI}$ vs. $\mathrm{AD} ;{ }^{\mathrm{c}} \mathrm{p}<0.05$ for $\mathrm{NC}$ vs. $\mathrm{MCI}, \mathrm{NC}$ vs. $\mathrm{AD}$ and $\mathrm{MCI}$ vs. $\mathrm{AD} ;{ }^{\mathrm{d}} \mathrm{p}<$ 0.001 for $\mathrm{NC}$ vs. $\mathrm{AD}$ and $\mathrm{MCI}$ vs. $\mathrm{AD} ;{ }^{\mathrm{e}} \mathrm{p}<0.01$ for $\mathrm{NC}$ vs. $\mathrm{MCI}$ and MCI vs. AD.

given analysis. A small number of subjects (13) showing a change from a more impaired diagnosis, MCI, to one less so, NC, were excluded from the primary analyses. The MCI to AD dementia progression analysis was later repeated including these 13 subjects, treated for the purposes of the analysis as stable MCI subjects, in order to make sure that the results were not substantially different. Occasional diagnosis instability or reversion is typical of this sort of MCI population. Subjects who remained stable at the specified baseline diagnosis were treated in the analysis as 'censored' observations, and the partial information they provided on time to change in diagnosis was used. Graphical and numerical checks on the assumption of proportional hazards were verified for all analyses. Where there was evidence of possible violation of the assumption, an additional nonparametric survival analysis was employed to verify and clarify the finding. Predictors were tested in a backward elimination algorithm with a cutoff for remaining in the model of $\mathrm{p} \leq 0.05$ (some marginal effects were allowed in). Backward elimination was used starting with baseline apathy, baseline anxiety, age, AMNART IQ, sex, APOE4 carrier status, Digit Symbol, RAVLT Total Learning and antidepressant use. Hallucinations were pre-excluded because all but one of the values for hallucinations were 0 across the sample of NC and MCI subjects.

This analysis was repeated with the Affective factor and Psychotic factor instead of the individual NPI-Q items.

\section{Results}

Table 1 displays demographic and clinical data for all subjects in the three diagnostic groups. There were significant differences in AMNART-IQ, MMSE, RAVLT
Total Learning, Digit Symbol, CDR-SB, APOE4 carrier status and antidepressant medication use between the $\mathrm{NC}, \mathrm{MCI}$ and $\mathrm{AD}$ dementia groups. The NC and AD dementia groups had a significantly smaller proportion of males than the MCI group did. Mean years of education for the NC and MCI groups were significantly higher than for the $\mathrm{AD}$ dementia group. Table 2 displays the presence (percent) of each of the 12 NPI-Q items for all subjects in the three diagnostic groups. The most common neuropsychiatric symptoms across all subjects were irritability, depression, anxiety, agitation, apathy and sleep, while the least common symptoms were hallucinations, euphoria and delusions. There were significant differences in depression, anxiety, apathy, disinhibition, irritability and appetite between the $\mathrm{NC}, \mathrm{MCI}$ and $\mathrm{AD}$ dementia groups. The NC and MCI groups had significantly fewer symptoms of delusions, hallucinations, aberrant motor behaviors and sleep when compared to the $\mathrm{AD}$ dementia group.

\section{Cross-Sectional Analysis: General Linear Model}

In the general linear regression model for all subjects, after backward elimination, greater hallucinations, anxiety and apathy severity were individually significantly associated with greater CDR-SB, representing global functional impairment (hallucinations: $\mathrm{p}<0.0001$; apathy: $\mathrm{p}<0.0001$; anxiety: $\mathrm{p}<0.0001$; table 3 ; fig. 1,2 ). The other NPI-Q items were not significantly associated with CDR-SB. Covariates that were significantly associated with CDR-SB were diagnostic group, sex (females > 
Table 2. Presence of neuropsychiatric symptoms in subjects at baseline

\begin{tabular}{lrcc}
\hline NPI-Q item & $\begin{array}{l}\text { NC } \\
(\mathrm{n}=229)\end{array}$ & $\begin{array}{l}\text { MCI } \\
(\mathrm{n}=395)\end{array}$ & $\begin{array}{l}\text { AD } \\
\text { dementia } \\
(\mathrm{n}=188)\end{array}$ \\
\hline Delusions & $0(0.0)^{\mathrm{d}}$ & $5(1.3)$ & $18(9.6)$ \\
Hallucinations & $1(0.4)^{\mathrm{d}}$ & $1(0.3)$ & $10(5.3)$ \\
Agitation & $6(2.6)^{\mathrm{e}}$ & $73(18.5)$ & $48(25.5)$ \\
Depression & $13(5.7)^{\mathrm{a}}$ & $76(19.2)$ & $64(34.0)$ \\
Euphoria & $0(0.0)^{\mathrm{f}}$ & $10(2.5)$ & $9(4.8)$ \\
Anxiety & $8(3.5)^{\mathrm{a}}$ & $71(18.0)$ & $65(34.6)$ \\
Apathy & $3(1.3)^{\mathrm{a}}$ & $55(13.9)$ & $64(34.0)$ \\
Disinhibition & $1(0.4)^{\mathrm{b}}$ & $30(7.6)$ & $34(18.1)$ \\
Irritability & $15(6.6)^{\mathrm{c}}$ & $109(27.6)$ & $70(37.2)$ \\
Aberrant motor behaviors & $1(0.4)^{\mathrm{d}}$ & $19(4.8)$ & $29(15.4)$ \\
Sleep & $21(9.2)^{\mathrm{d}}$ & $46(11.6)$ & $49(26.1)$ \\
Appetite & $1(0.4)^{\mathrm{c}}$ & $45(11.4)$ & $33(17.6)$ \\
\hline
\end{tabular}

All values represent numbers of subjects (percentage in parentheses). ${ }^{a} \mathrm{p}<0.0001$ for NC vs. MCI, NC vs. AD and MCI vs. AD; ${ }^{b} \mathrm{p}<0.01$ for $\mathrm{NC}$ vs. MCI, NC vs. AD and MCI vs. AD; ${ }^{c} \mathrm{p} \leq 0.05$ for $\mathrm{NC}$ vs. MCI, NC vs. AD and MCI vs. $A D ;{ }^{\mathrm{d}} \mathrm{p}<0.001$ for $\mathrm{NC}$ vs. $\mathrm{AD}$ and $\mathrm{MCI}$ vs. $\mathrm{AD} ;{ }^{\mathrm{e}} \mathrm{p}<0.0001$ for $\mathrm{NC}$ vs. MCI and NC vs. AD; ${ }^{\mathrm{f}} \mathrm{p}<0.01$ for NC vs. AD.

males), age (quadratic term), duration of AD symptoms, RAVLT Total Learning and Digit Symbol (all in expected directions; $\mathrm{R}^{2}=0.77, \mathrm{p}<0.0001$ for overall model; table $3)$. None of the interactions of diagnostic group with NPI-Q items were significant, indicating that the relation of hallucinations, anxiety and apathy to CDR-SB was not conditional on the diagnostic group. The diagnostic group uniquely accounted for $11 \%$ of the total variance in CDR-SB, while all the other significant predictors each individually accounted for $1 \%$ or less of the total variance. Residual distributions reasonably conformed to model assumptions and indicated good model fit.

\section{Longitudinal Analyses: Mixed Effects Model}

Informed by the results of the cross-sectional analysis above, we assessed the longitudinal relationship between the significant NPI-Q items (hallucinations, anxiety and apathy) and CDR-SB. In the mixed random and fixed coefficient longitudinal regression model for all subjects, after backward elimination, greater baseline hallucinations and apathy severity were significantly associated with a greater rate of increase in CDR-SB over time (hallucinations: $\mathrm{p}<0.0001$; apathy: $\mathrm{p}=0.04$; table 4 ; fig. 3 , 4). Additional significant fixed effect predictors were interaction of baseline CDR-SB with time and interaction of diagnosis with time, age, RAVLT Total Learning and Digit Symbol (all in expected directions; $\mathrm{R}^{2}=0.69, \mathrm{p}<$ 0.0001 for overall model fixed effects; $\mathrm{R}^{2}=0.94$ including random terms, $\mathrm{p}<0.0001$; table 4$)$. There was significant random variation in slope and intercepts of time trajectories across subjects and no correlation between the two.

When using the Affective factor and Psychotic factor instead of the individual NPI-Q items, we found that a greater baseline Psychotic factor score was significantly associated with a greater rate of increase in CDR-SB over time $(\mathrm{p}<0.0001)$. Additional significant fixed effect predictors were interaction of baseline CDR-SB with time and interaction of diagnosis with time, RAVLT Total Learning, Digit Symbol, Affective factor and age (all in expected directions; $\mathrm{R}^{2}=0.69, \mathrm{p}<0.0001$ for overall model fixed effects; $\mathrm{R}^{2}=0.94$ including random terms, $\mathrm{p}<0.0001$ ).

Longitudinal Analyses: Cox Proportional Hazards Model

Eleven out of 223 subjects (4.9\%) with a baseline diagnosis of NC progressed to a diagnosis of MCI over the 3 -year follow-up period (212 subjects were censored). One hundred and fifty-six out of 365 subjects (42.7\%) with a baseline diagnosis of MCI progressed to a diagnosis of AD dementia over the 3-year follow-up period (209 subjects were censored).

\section{MCI to AD Dementia Progression Predicted with}

NPI-Q Items

Greater baseline anxiety showed greater hazard for change from $\mathrm{MCI}$ to $\mathrm{AD}$ dementia [hazard ratio $(\mathrm{HR})=$ $1.33,95 \%$ CI 1.03-1.68; $p=0.02$; fig. 5].

Here the hazard rate is the probability of changing to the more impaired diagnosis at a given time among those who have not already done so. The hazard ratio is the ratio of this rate relative to the rate for a stated reference level or for a change in a given predictor of 1 unit or an otherwise specified number of units.

Other predictors showed significant effects as well; hazard increased with a greater number of APOE4 alleles $(\mathrm{HR}=1.58,95 \% \mathrm{CI} 1.26-1.97 ; \mathrm{p}<0.0001)$ and lower baseline scores on Digit Symbol (HR $=0.98,95 \%$ CI 0.96-0.99; $\mathrm{p}=0.003)$ and RAVLT Total Learning $(\mathrm{HR}=0.92,95 \% \mathrm{CI}$ $0.90-0.94 ; \mathrm{p}<0.0001)$, and females showed greater hazard than males ( $\mathrm{HR}=1.74,95 \%$ CI 1.25-2.42; $\mathrm{p}=0.001)$.

This analysis was repeated after including the $13 \mathrm{sub}-$ jects who improved from MCI to NC, treated as stable MCI subjects for purposes of the analysis, and the results were virtually the same (data not shown).

When using the Affective factor and Psychotic factor instead of the individual NPI-Q items, we found the fol- 
Table 3. Cross-sectional general linear model of association of NPI-Q items and CDR-SB, displaying predictors retained in the final model (model: $\mathrm{R}^{2}=0.77, \mathrm{p}<0.0001$ )

\begin{tabular}{|c|c|c|c|c|c|}
\hline \multirow[t]{2}{*}{ Predictor } & \multirow[t]{2}{*}{$\beta$} & \multirow[t]{2}{*}{$95 \%$ CI for $\beta$} & \multirow[t]{2}{*}{$\mathrm{p}$} & \multicolumn{2}{|c|}{$\begin{array}{l}\text { Percent variance } \\
\text { accounted for }\end{array}$} \\
\hline & & & & total & partial \\
\hline Apathy & 0.41 & 0.29 to 0.52 & $<0.0001$ & 1.4 & 5.4 \\
\hline Hallucinations & 0.94 & 0.47 to 1.41 & $<0.0001$ & 0.4 & 1.8 \\
\hline Anxiety & 0.26 & 0.14 to 0.38 & $<0.0001$ & 0.5 & 2.1 \\
\hline \multicolumn{6}{|l|}{ Diagnosis $^{1}$} \\
\hline $\mathrm{AD}$ & 3.42 & 0.11 & $<0.0001$ & 11.0 & 31.7 \\
\hline MCI & 1.76 & 0.05 & & & \\
\hline $\mathrm{NC}$ & 0.55 & 0.08 & & & \\
\hline AD symptom duration & 0.18 & 0.12 to 0.23 & $<0.0001$ & 1.2 & 4.8 \\
\hline Digit Symbol & -0.01 & -0.02 to -0.006 & $<0.0001$ & 0.4 & 1.8 \\
\hline \multicolumn{6}{|l|}{ Age } \\
\hline Quadratic term & -0.002 & 0.0006 to 0.003 & 0.001 & 0.3 & 1.1 \\
\hline Linear term & -0.24 & -0.38 to -0.09 & 0.001 & 0.3 & 1.1 \\
\hline \multicolumn{6}{|l|}{$\operatorname{Sex}^{1}$} \\
\hline Female & 2.01 & 0.05 & 0.004 & 0.2 & 0.9 \\
\hline Male & 1.82 & 0.05 & & & \\
\hline RAVLT Total Learning & -0.01 & -0.02 to -0.003 & 0.009 & 0.2 & 0.7 \\
\hline
\end{tabular}

\footnotetext{
'Percent variance total' represents the percentage of total variance of CDR-SB uniquely associated with the indicated predictor (unbiased population estimate). 'Percent variance partial' represents the percentage of variance of CDR-SB, with the portion associated with other predictors previously removed, which is uniquely associated with the indicated predictor (unbiased population estimate). $\beta=$ Partial unstandardized regression coefficient estimate.

${ }^{1}$ For diagnosis and sex, covariate adjusted means and standard errors are reported instead of $\beta$ and $95 \% \mathrm{CI}$ for $\beta$.
}

lowing: a greater baseline Psychotic factor score showed greater hazard for change from MCI to AD dementia $(\mathrm{HR}=1.53,95 \% \mathrm{CI} 1.19-1.91 ; \mathrm{p}=0.0003)$. Other predictors showed significant effects as well, i.e. sex (females $>$ males), APOE4 carrier status, RAVLT Total Learning and Digit Symbol (all in expected directions).

NC to MCI Progression Predicted with NPI-Q Items

None of the neuropsychiatric symptoms were retained in the final model. Only one significant predictor was retained, namely baseline Digit Symbol, with lower scores associated with greater hazard for change from $\mathrm{NC}$ to MCI (HR $=0.93$, 95\% CI 0.87-0.99; $\mathrm{p}=0.02)$.

\section{Discussion}

Individual and cumulative neuropsychiatric symptoms impose a heavy caregiver burden in MCI and $\mathrm{AD}$ dementia and, along with impairments in $\mathrm{ADL}$, are major causes of nursing home placement $[5,9,10,23]$. The results of the current study suggest that hallucinations, apathy and anxiety, but no other neuropsychiatric symptoms assessed at baseline, are associated with greater baseline global functional impairment across the continuum of $\mathrm{AD}$. This adds to prior research identifying neuropsychiatric symptoms, among others, as cross-sectionally related to global functional impairment [20]. Observing this relationship, independent of diagnosis, age, sex, duration of AD symptoms, premorbid intelligence, APOE4 carrier status, memory performance, processing speed and psychotropic medication use, in a large, well-characterized cohort builds on prior findings and suggests that these three neuropsychiatric symptoms, in particular, are especially important and useful when exploring proxies of global functioning in $\mathrm{AD}$.

The findings of this study are consistent with the findings of a large cross-sectional nationally representative cohort study [20] with subjects ranging from conditions of normal cognition to severe dementia, in which 


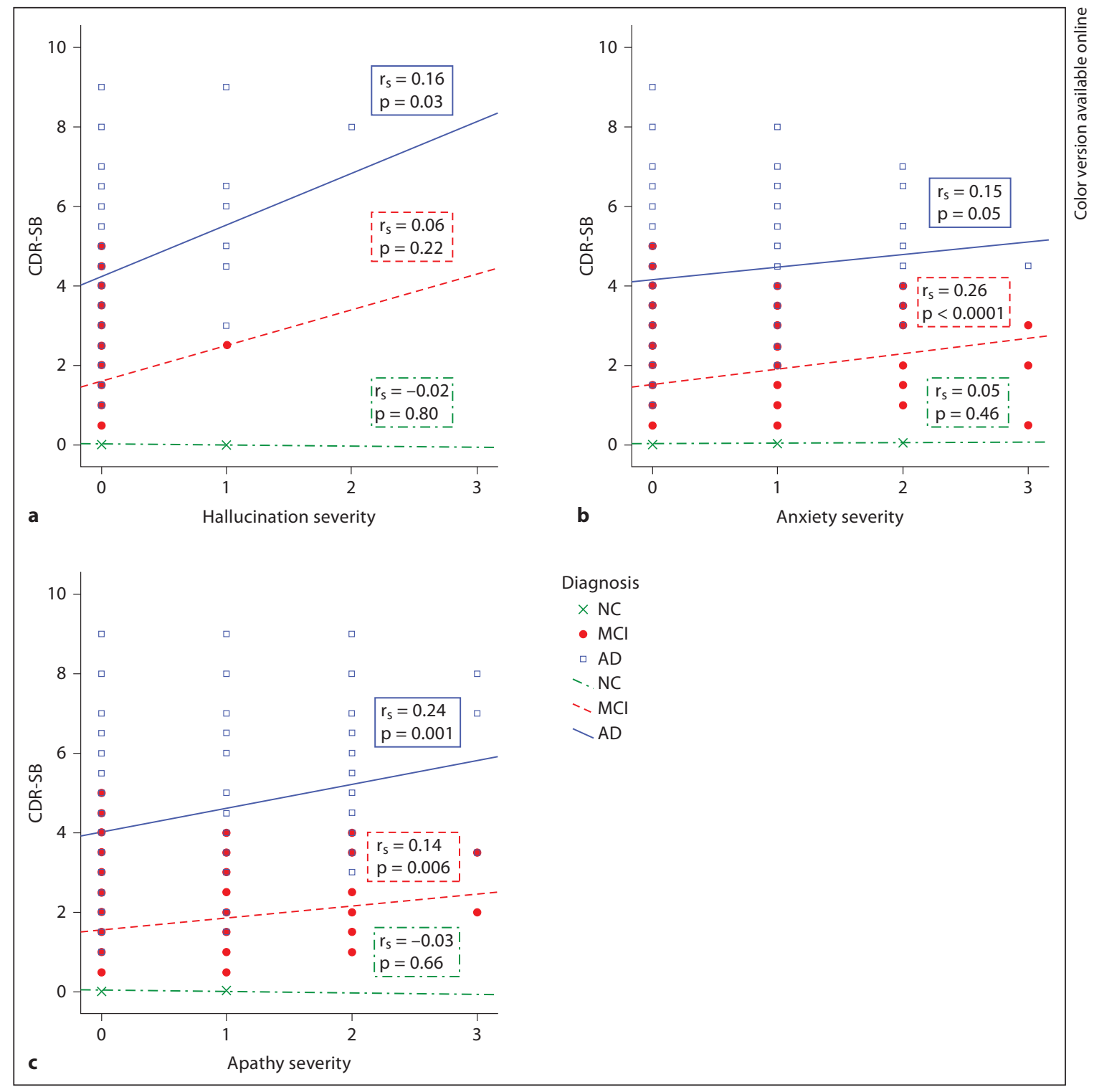

Fig. 1. Scatter plots of CDR-SB, representing global functional impairment, vs. the severity of the NPI-Q items hallucinations (a), anxiety (b) and apathy (c) in NC, MCI and AD dementia subjects. Spearman's rank correlation coefficients $\left(r_{s}\right)$ and corresponding $\mathrm{p}$ values are provided for each diagnostic group. These graphs represent univariate unadjusted results.

investigators found that clinically significant depression was associated with greater basic and instrumental ADL impairment, anxiety and aberrant motor behaviors were associated with greater instrumental ADL impairment, and hallucinations and apathy showed a trend toward greater basic ADL impairment; that study also showed that the presence of 3 or more neuropsychiatric symptoms or 1 neuropsychiatric symptom scored as clinically significant, while controlling for covariates including diagnostic group, was associated with greater basic and instrumental ADL impairment [20]. These data highlight both the cumulative and individual effects of neuropsychiatric symptoms such as depression, anxiety, aberrant motor behavior and, to a lesser extent, hallucinations and apathy on functional impairment.

Building on these cross-sectional results, we sought to determine if baseline individual neuropsychiatric symptoms are related to longitudinal change in global functional impairment. We found that increased hallucinations and apathy at baseline were each significantly 


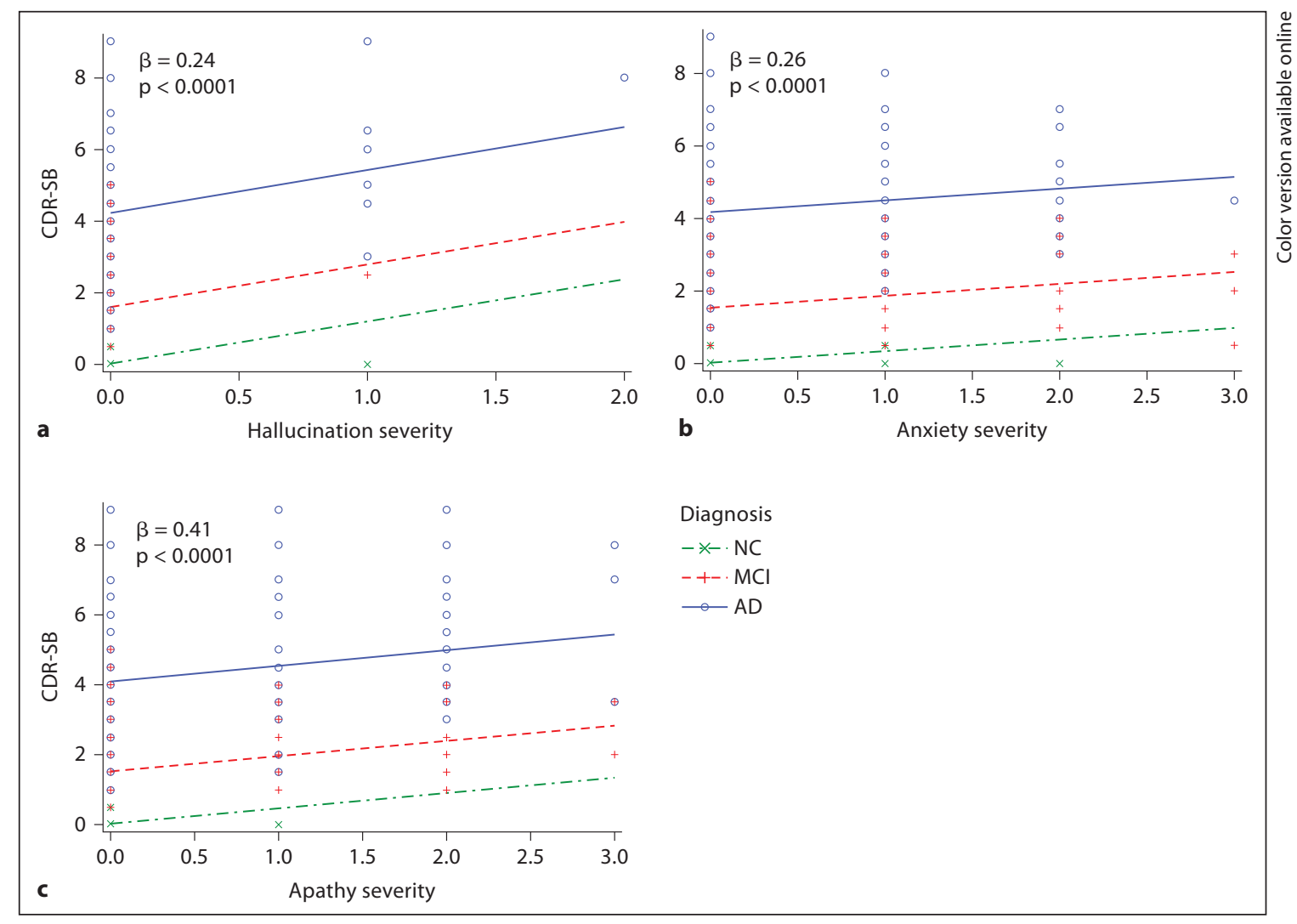

Fig. 2. Values predicted from a general linear model of CDR-SB regressed on diagnostic group and the severity of the NPI-Q items hallucinations $(\mathbf{a})$, anxiety (b) and apathy $(\mathbf{c})$. The lines indicate the predicted values for CDR-SB, and the symbols denote corresponding actual values (overlapping observations at the same coordinates are sometimes hidden). The final model included a number of additional partialed significant predictors, but to simplify the visual display, they were not included in the model producing the predicted values in the figures (including them had a negligible effect on the relations seen). $\beta=$ Partial regression coefficient estimate.

associated with worsening global functional impairment over time across the AD spectrum, independent of many potential confounders. This is consistent with other longitudinal analyses, which have also shown an association between hallucinations and global functional decline $[23,24,38]$. Although Tschanz et al. [3] found only weak correlations between total NPI score and CDR-SB score, they suggest that global scores may have obscured specific neuropsychiatric symptom and clinical correlations; in addition, their findings reinforced the increasing prevalence and persistence of apathy into late-stage dementia. As such, we chose to focus on individual neuropsychiatric symptoms in order to determine their relationship with proxies of disease progression, such as longitudinal CDR-SB. Moreover, since our study population consisted of the early $\mathrm{AD}$ spectrum in which neuropsychiatric symptom prevalence and severity are lower, we did not want to obscure the potential importance of individual symptoms by solely reporting an association with total NPI-Q score or clusters of symptoms.

Consistent with our findings of an association between hallucinations and global functional decline over time, an early prospective study of 177 individuals with probable or possible $\mathrm{AD}$ showed accelerated cognitive decline in a subgroup of 30 subjects with visual or auditory hallucinations independent of baseline cognitive function and neuroleptic exposure [39]. Subsequently, a large multicenter prospective cohort of subjects with early $\mathrm{AD}$ followed for 4.5 years demonstrated increased risk of cognitive and functional decline with the presence of both delusions and hallucinations and elevated risks of institutionalization and death associated with halluci- 
Table 4. Longitudinal mixed effects model of association of baseline NPI-Q items and CDR-SB over time, displaying predictors retained in the final model (model: $\mathrm{R}^{2}=0.69$ for fixed effects, $\mathrm{p}<$ $0.0001 ; \mathrm{R}^{2}=0.94$ including random terms, $\mathrm{p}<0.0001$ )

\begin{tabular}{lccc}
\hline Predictor & $\beta$ & $95 \%$ CI for $\beta$ & $\mathrm{p}$ \\
\hline $\begin{array}{l}\text { Time } \\
\text { Baseline hallucinations } \times\end{array}$ & 0.11 & -0.02 to 0.24 & $<0.0001$ \\
$\quad$ time & 0.17 & 0.01 to 0.32 & 0.04 \\
$\begin{array}{l}\text { Baseline apathy } \times \text { time } \\
\text { Baseline CDR-SB } \times \text { time }\end{array}$ & 0.18 & 0.09 to 0.26 & $<0.0001$ \\
$\begin{array}{l}\text { Baseline diagnosis } \times \text { time } \\
\quad \text { AD }\end{array}$ & 0.87 & 0.45 to 1.29 & 0.0002 \\
$\quad$ MCI & 0.36 & 0.15 to 0.57 & \\
$\quad$ NC & 0 & & $<0.0001$ \\
$\begin{array}{l}\text { Baseline RAVLT Total } \\
\quad \text { Learning }\end{array}$ & -0.02 & -0.03 to -0.009 & $<0.0001$ \\
$\begin{array}{l}\text { Baseline Digit Symbol } \\
\text { Baseline age }\end{array}$ & -0.009 & -0.02 to -0.003 & 0.007 \\
& 0.01 & -0.0004 to 0.02 & 0.06 \\
\hline
\end{tabular}

The symbol $\times$ indicates an interaction. $\beta=$ Partial regression coefficient estimate.

nations [23]. The same group later found that disruptive behavior in $\mathrm{AD}$ was also associated with increased risk of cognitive and functional decline and institutionalization [22]. These and other studies have also highlighted hallucinations as a poor prognostic factor in $\mathrm{AD}[24,40]$.

A few studies have looked for localization of hallucinations separate from delusions in the brain of patients with $\mathrm{AD}$ dementia and have found an association with left dorsolateral prefrontal, right parietal and left medial temporal hypoperfusion and occipital atrophy [41, 42]. These associations suggest a specific neurodegenerative process disrupting the neural system when hallucinations manifest in $\mathrm{AD}$.

A 2-year longitudinal study of subjects with mild to moderate $\mathrm{AD}$ dementia demonstrated an increased risk of basic ADL decline in subjects characterized as having an affective syndrome (anxiety and depression) and an increased risk of cognitive decline with a manic syndrome (euphoria and disinhibition) [21]. These results are different from our findings, but our study focused on the CDR-SB as the dependent variable, which combines cognitive and ADL items, examined individual neuropsychiatric symptoms rather than syndromes and consisted of subjects across the AD spectrum, earlier on, from NC to mild AD dementia.

A 3-year prospective study of MCI outpatients demonstrated a 7-fold increased risk of progression to $\mathrm{AD}$ dementia with elevated scores on apathy measures but no increased risk in patients with symptoms of depression [14]. Here, we show an association between greater baseline apathy and worsening global functioning. Apathy has been associated with reduced activity (hypoperfusion or hypometabolism suggesting synaptic dysfunction) in the anterior cingulate, orbitofrontal and right temporoparietal cortices in AD dementia [43-47]. Similarly, downstream disease manifestations, including increased neurofibrillary tangle burden in the anterior cingulate at postmortem, increased cerebrospinal fluid total and phospho-tau, and medial frontal atrophy visualized with structural magnetic resonance imaging, have been associated with greater apathy in AD dementia [48-50]. These findings, reproduced in several modalities, demonstrate a specific neurodegenerative process disrupting a neural circuit involving the medial frontal regions and further strengthen the evidence for apathy being as intrinsic to $\mathrm{AD}$ as are cognitive and functional decline.

Although the overall effects of neuropsychiatric symptoms in our longitudinal model were small when compared to other predictors, such as diagnostic group, they were significant independent of diagnostic group, cognitive impairment and common demographic predictors. Therefore, the clinical implication of our findings is that older individuals experiencing the neuropsychiatric symptoms of apathy or hallucinations at baseline, even prior to developing mild dementia, should be monitored closely as they have a greater likelihood of future global functional impairment than those not experiencing either or both of these neuropsychiatric symptoms.

We further sought to determine whether neuropsychiatric symptoms had the ability to predict disease progression, specifically progression from NC to MCI and from $\mathrm{MCI}$ to $\mathrm{AD}$ dementia. Over a period of 3 years, a little over $40 \%$ of MCI subjects progressed to $\mathrm{AD}$ dementia, consistent with prior studies of amnestic MCI [51, 52]. Furthermore, we found that increased anxiety at baseline was associated with increased hazard of progression from MCI to AD dementia, again independent of many potential confounders. These results suggest that individuals with anxiety at baseline are likely to decline faster and progress to dementia compared to those without anxiety. This finding is in agreement with other studies demonstrating that anxiety is a risk factor for progression from MCI to dementia. The role of anxiety as an earlystage behavioral symptom in $\mathrm{AD}$ was investigated in a 3 -year population-based study of 47 elderly participants with MCI; the study found that anxiety, but not depressive symptoms, was strongly predictive of progression to 
Fig. 3. Predicted values from fixed effects of a best-fitting longitudinal model of CDR-SB by the NPI-Q item hallucinations and selected baselines according to diagnostic group. a NC. b MCI. c AD dementia. Age, NPI-Q apathy, RAVLT Total Learning and Digit Symbol at baseline were set equal to grand means.

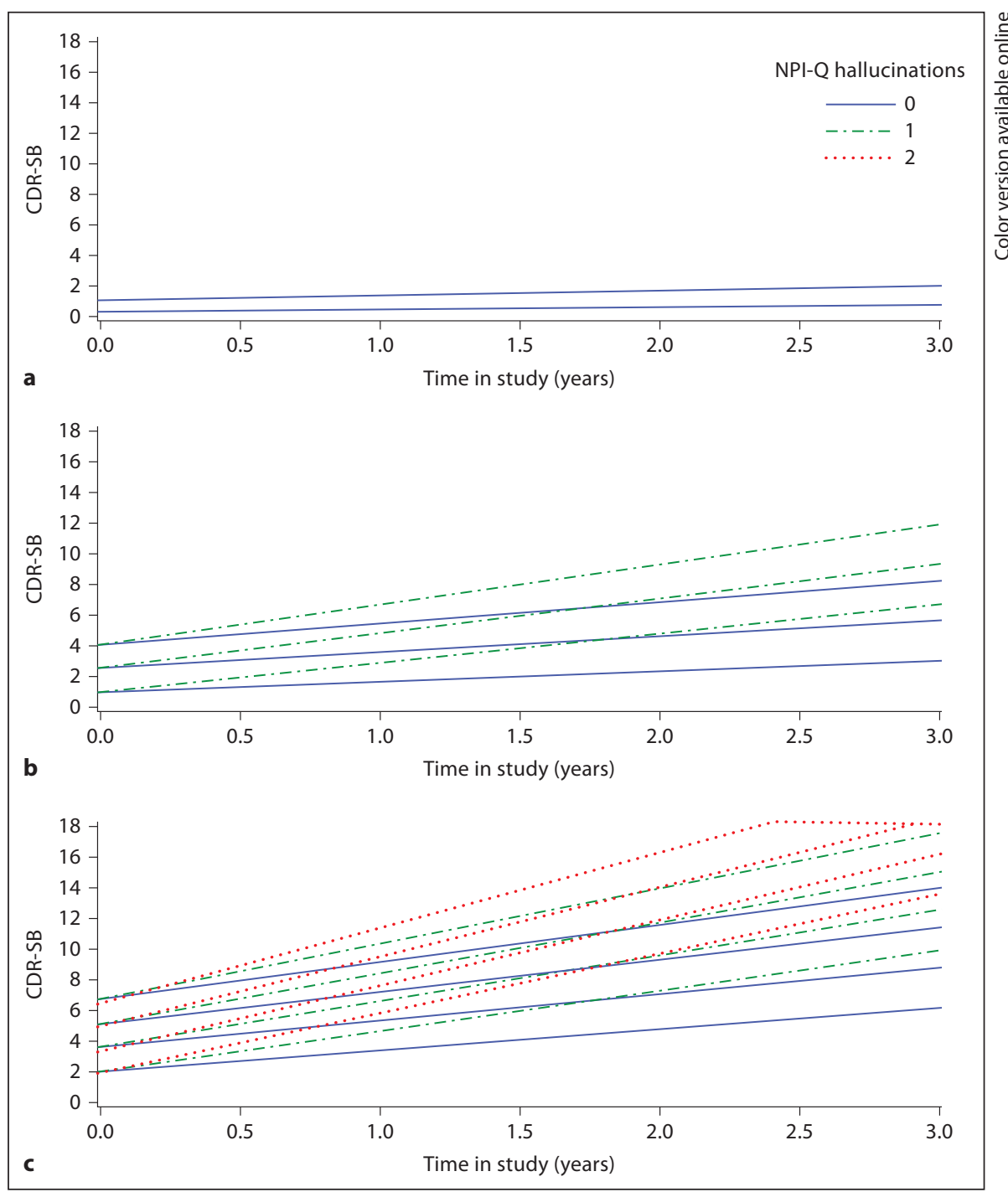

dementia, with a doubling of risk for increased anxiety symptoms [13].

Unlike previous reports [14], we did not see an association between increased apathy at baseline and progression from MCI to AD dementia. These results also do not align with our longitudinal assessment of global functional impairment, which did show an association with apathy. It is unclear why different neuropsychiatric symptoms were associated with disease progression assessed by change in diagnosis over time as opposed to global functional decline over time. It is possible that the sample population and sample size may have accounted for these findings; the Cox proportional hazards model only included MCI subjects, while the longitudinal mixed effects model included NC and AD dementia subjects as well. Moreover, although the previous study quoted [14] accounted for many of the same covariates our study did, we had nearly 3 times as many subjects. Our Cox proportional hazards findings further confirmed previous reports that having one or more APOE4 allele, greater memory impairment or slower processing speed at baseline increased the risk of progression from $\mathrm{MCI}$ to $\mathrm{AD}$ dementia [51, 53, 54].

We did not see a significant association between individual neuropsychiatric symptoms and progression from NC to MCI. This is likely due to the very small number of subjects who progressed to MCI (5\%) and the low frequency of baseline neuropsychiatric symptoms in our NC subjects. A longer follow-up period and/or an assessment of an increase in neuropsychiatric symptoms over 
Fig. 4. Predicted values from fixed effects of a best-fitting longitudinal model of CDR-SB by the NPI-Q item apathy and selected baselines according to diagnostic group. a NC. b MCI. c AD dementia. Age, NPI-Q hallucinations, RAVLT Total Learning and Digit Symbol at baseline were set equal to grand means.

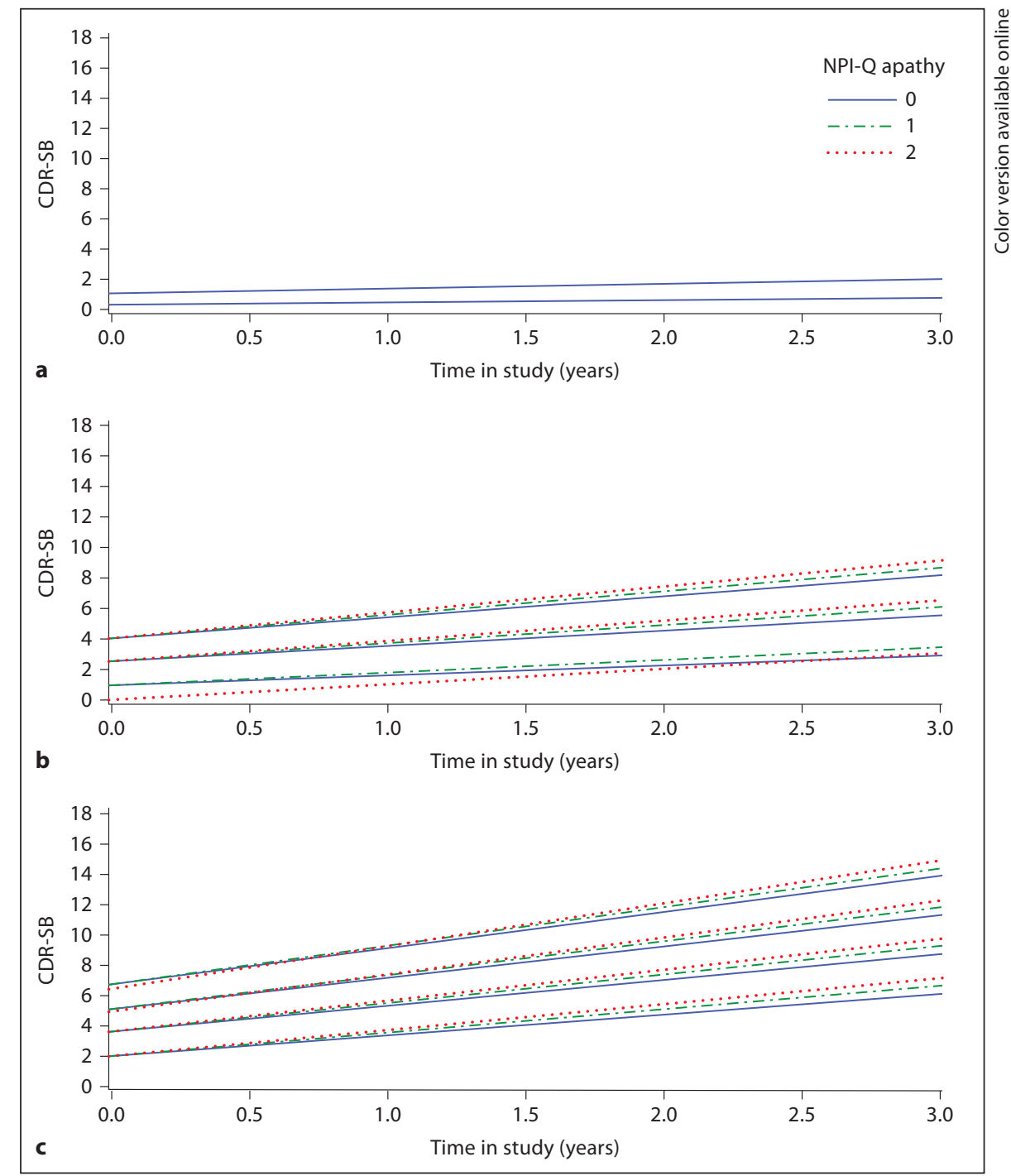

time or new development of neuropsychiatric symptoms in NC subjects will help further determine the potential of neuropsychiatric symptoms to influence progression from NC to MCI.

Interestingly, hallucinations, which were the least common neuropsychiatric symptom in this sample (occurring in $1.5 \%$ of subjects), were significantly associated with greater global functional impairment cross-sectionally and longitudinally, while other common neuropsychiatric symptoms were not. For example, the two most common neuropsychiatric symptoms in the cohort, irritability (occurring in $24 \%$ of subjects) and depression (19\%), were not significantly associated with global functional impairment. This is reflected in prior studies showing an inconsistent association between depression and progression from MCI to dementia despite its high prevalence $[13,14,55-57]$. Another consideration is that although hallucinations are rare, they represent a likely sign of neurodegenerative disease in the elderly in the absence of confounding conditions such as significant cerebrovascular disease, infections or medication side effects. On the other hand, depression and irritability, which are common in the elderly, may not be as specific to neurodegeneration.

Prior studies have performed factor analyses to define neuropsychiatric symptom clusters, which may reflect shared pathophysiology or underlying regional brain changes $[15,58]$. These studies looked at individuals at the stage of dementia, while our study included less impaired individuals. For that reason, in our main 


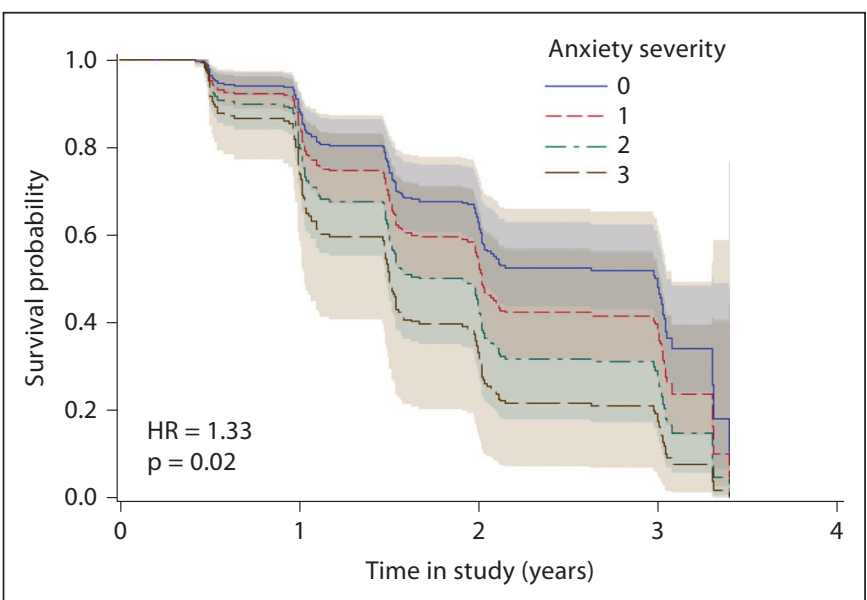

Fig. 5. Kaplan-Meier survival curves of progression from MCI to $\mathrm{AD}$ dementia as predicted by the NPI-Q item anxiety, where 'survival' stands for absence of progression from MCI to AD dementia as yet. Predicted values displayed are estimated holding other significant covariates in the model (baseline RAVLT Total Learning, baseline Digit Symbol, APOE4 status and sex) constant at their respective grand means, and setting sex as female. Shading indicates $95 \%$ confidence limits.

analyses we used individual neuropsychiatric symptoms. However, we subsequently performed a factor analysis of the 12 NPI-Q items and came up with 2 factors (clusters of symptoms), an Affective factor and a Psychotic factor. Prior studies have reported more factors (up to 5), again possibly because of a more impaired population with better defined and more severe neuropsychiatric syndromes $[15,58]$.

We then repeated our longitudinal analyses using the factors instead of the individual neuropsychiatric symptoms. The Psychotic factor, but not the Affective factor, was significantly associated with disease progression (worsening CDR-SB over time and progression from $\mathrm{MCI}$ to AD dementia). These results were a little different from our original results using the individual neuropsychiatric symptoms. The Psychotic factor strongly correlated with hallucinations, and therefore it is not surprising that similar to the model employing hallucinations, it showed a significant association with CDR-SB over time. While the Affective factor had a strong correlation with apathy, it was composed of many more neuropsychiatric symptoms, none of which (with the exception of anxiety) were associated with CDR-SB in the original analyses, which could have led to a weaker association of the Affective factor with CDR-SB over time. Moreover, the association of apathy with CDR-SB over time was not as strong as that of hallucinations, again potentially leading to a weaker association of the Affective factor with CDR-SB over time. Finally, in the original model predicting progression from MCI to $\mathrm{AD}$ dementia, anxiety was associated with progression, while in the new model using the factors, the Psychotic factor was associated with progression. Even though the Psychotic factor did not include anxiety, it was correlated with it. Moreover, anxiety did load onto the Psychotic factor but not as strongly as it did onto the Affective factor, which led to anxiety ultimately being a part of the Affective factor. Therefore, it is possible that shared variance between anxiety and the Psychotic factor was contributing to the association with progression from MCI to AD dementia.

There were several limitations to this study. Firstly, the ADNI screening protocol was very selective and excluded subjects with active primary psychiatric disorders, which often co-occur with $\mathrm{MCI}$ and $\mathrm{AD}$ dementia, and therefore limited the prevalence of many neuropsychiatric symptoms. Moreover, the $\mathrm{AD}$ dementia group was only mildly impaired at baseline (MMSE $23.3 \pm 2.0$ ), further reducing the occurrence and severity of neuropsychiatric symptoms. Thus, this sample might not be highly representative of patients in clinical practice. That said, this study population provided us with a unique opportunity to longitudinally study neuropsychiatric symptoms in the early $\mathrm{AD}$ spectrum, which has not been done as much in the literature and follows a recent trend in the AD field to move toward earlier diagnosis. Secondly, the average years of education $(15.5 \pm 3.1)$ and AMNART IQ score (117.2 \pm 11.6$)$, representing premorbid intelligence, indicate that this sample is more educated than the average population, again reducing the generalizability of the study. However, we controlled for AMNART IQ scores in all of our analyses. Moreover, while this is a highly selected and carefully characterized population, it does represent the population typically seen in clinical trials in the $\mathrm{AD}$ spectrum. Therefore, these results can be reliably related to such a setting and provide further evidence for the importance of assessing neuropsychiatric symptoms in clinical trials. Thirdly, the NPI-Q was the only available scale in the ADNI database that examined multiple neuropsychiatric symptoms. This scale uses one question to assess the presence or absence of each symptom, which is followed by a severity rating. There are other more specialized and comprehensive scales used in the assessment of individual neuropsychiatric symptoms or syndromes, which can provide richer and potentially more valid information. However, the NPI-Q has been used widely in clinical trials and can be useful as a quick 
screening test clinically. Fourthly, we did not account for use of approved $\mathrm{AD}$ medications, such as cholinesterase inhibitors, and it is likely that many of the AD dementia subjects and some of the MCI subjects were taking such medications. On the other hand, we did account for the use of common psychiatric medications; antidepressant use was noted in about 1 out of every 5 subjects and was included as a covariate, while other psychiatric medications were used by less than $2 \%$ of subjects and therefore were not included as covariates. Fifthly, some of the effects are significant but do not have large effect sizes as reflected by the percent variance accounted for by some of the individual predictors and the partial regression coefficients. This is due to the large sample size of this study, which allows small effects to remain significant. We therefore reported estimates of effect size where possible. Finally, a possible limitation is the focus on individual neuropsychiatric symptoms rather than neuropsychiatric symptom clusters or syndromes, as has been used by some groups, because certain neuropsychiatric symptoms may be highly intercorrelated in AD dementia. However, most recent studies examining functional outcomes have continued to use individual neuropsychiatric symptoms as predictor variables, as we did in the main analyses of our study $[3,20,23,24]$. This approach allows for more direct comparisons between studies until a clear consensus emerges regarding the distribution and number of neuropsychiatric symptoms which belong within each cluster. Therefore, we wanted to take advantage of this large, longitudinal dataset with a range of disease severity across the $\mathrm{AD}$ spectrum to determine which individual neuropsychiatric symptoms drive global functional decline over time. We then performed a factor analysis and looked at clusters of symptoms reproducing some but not all of our results, further justifying our emphasis on individual neuropsychiatric symptoms in our mildly impaired study population.
In conclusion, the neuropsychiatric symptoms of apathy and hallucinations were associated with current and future global functional impairment in our sample of NC, MCI and mild AD dementia subjects. Future studies incorporating imaging and cerebrospinal fluid biomarkers may serve to elucidate the underlying pathophysiology associated with these symptoms.

\section{Acknowledgements}

This study was supported by grants R01 AG027435S, K23 AG033634 and K24 AG035007, the Rosalinde and Arthur Gilbert Foundation/American Federation for Aging Research New Investigator Awards in Alzheimer's Disease, the Massachusetts Alzheimer's Disease Research Center (P50 AG005134), the Harvard Aging Brain Study (P01 AGO36694) and the ADNI (National Institutes of Health grant U01 AG024904). The ADNI is funded by the National Institute on Aging, the National Institute of Biomedical Imaging and Bioengineering and through generous contributions from the following: Abbott, AstraZeneca AB, Bayer Schering Pharma AG, Bristol-Myers Squibb, Eisai Global Clinical Development, Elan Corporation, Genentech, GE Healthcare, GlaxoSmithKline, Innogenetics, Johnson and Johnson, Eli Lilly and Co., Medpace Inc., Merck and Co. Inc., Novartis AG, Pfizer Inc., F. Hoffman-La Roche, Schering-Plough and Synarc Inc., as well as the nonprofit partners the Alzheimer's Association and Alzheimer's Drug Discovery Foundation, with participation from the US Food and Drug Administration. Private sector contributions to ADNI are facilitated by the Foundation for the National Institutes of Health (www.fnih. org). The grantee organization is the Northern California Institute for Research and Education, and the study is coordinated by the Alzheimer's Disease Cooperative Study at the University of California, San Diego. ADNI data are disseminated by the Laboratory for Neuro Imaging at the University of California, Los Angeles. This research was also supported by National Institutes of Health grants P30 AG010129 and K01 AG030514 and the Dana Foundation.

\section{References}

1 Lyketsos CG, Lopez O, Jones B, Fitzpatrick AL, Breitner J, DeKosky S: Prevalence of neuropsychiatric symptoms in dementia and mild cognitive impairment: results from the cardiovascular health study. JAMA 2002;288: 1475-1483.

-2 Apostolova LG, Cummings JL: Neuropsychiatric manifestations in mild cognitive impairment: a systematic review of the literature. Dement Geriatr Cogn Disord 2008;25: 115-126.
3 Tschanz JT, Corcoran CD, Schwartz S, Treiber K, Green RC, Norton MC, Mielke MM, Piercy K, Steinberg M, Rabins PV, Leoutsakos JM, Welsh-Bohmer KA, Breitner JC, Lyketsos CG: Progression of cognitive, functional, and neuropsychiatric symptom domains in a population cohort with Alzheimer's dementia: the Cache County Dementia Progression Study. Am J Geriatr Psychiatry 2011;19:532-542.
4 Mohamed S, Rosenheck R, Lyketsos CG, Schneider LS: Caregiver burden in Alzheimer disease: cross-sectional and longitudinal patient correlates. Am J Geriatr Psychiatry 2010;18:917-927.

5 Okura T, Langa KM: Caregiver burden and neuropsychiatric symptoms in older adults with cognitive impairment: the Aging, Demographics, and Memory Study (ADAMS). Alzheimer Dis Assoc Disord 2011;25:116-121. 
-6 Shin IS, Carter M, Masterman D, Fairbanks L, Cummings JL: Neuropsychiatric symptoms and quality of life in Alzheimer disease. Am J Geriatr Psychiatry 2005;13:469-474.

7 Murman DL, Chen Q, Powell MC, Kuo SB, Bradley CJ, Colenda CC: The incremental direct costs associated with behavioral symptoms in AD. Neurology 2002;59:1721-1729.

-8 Beeri MS, Werner P, Davidson M, Noy S: The cost of behavioral and psychological symptoms of dementia (BPSD) in community dwelling Alzheimer's disease patients. Int J Geriatr Psychiatry 2002;17:403-408.

-9 Steele C, Rovner B, Chase GA, Folstein M: Psychiatric symptoms and nursing home placement of patients with Alzheimer's disease. Am J Psychiatry 1990;147:1049-1051.

$\checkmark 10$ Yaffe K, Fox P, Newcomer R, Sands L, Lindquist K, Dane K, Covinsky KE: Patient and caregiver characteristics and nursing home placement in patients with dementia. JAMA 2002;287:2090-2097.

- 11 McKhann GM, Knopman DS, Chertkow H, Hyman BT, Jack CR Jr, Kawas CH, Klunk WE, Koroshetz WJ, Manly JJ, Mayeux R, Mohs RC, Morris JC, Rossor MN, Scheltens P, Carrillo MC, Thies B, Weintraub S, Phelps CH: The diagnosis of dementia due to Alzheimer's disease: recommendations from the National Institute on Aging-Alzheimer's Association workgroups on diagnostic guidelines for Alzheimer's disease. Alzheimers Dement 2011;7:263-269.

-12 Monastero R, Mangialasche F, Camarda C, Ercolani S, Camarda R: A systematic review of neuropsychiatric symptoms in mild cognitive impairment. J Alzheimers Dis 2009;18: 11-30.

13 Palmer K, Berger AK, Monastero R, Winblad B, Backman L, Fratiglioni L: Predictors of progression from mild cognitive impairment to Alzheimer disease. Neurology 2007;68: 1596-1602.

- 14 Palmer K, Di Iulio F, Varsi AE, Gianni W, Sancesario G, Caltagirone C, Spalletta G: Neuropsychiatric predictors of progression from amnestic-mild cognitive impairment to Alzheimer's disease: the role of depression and apathy. J Alzheimers Dis 2010;20: 175-183.

15 Aalten P, Verhey FR, Boziki M, Bullock R, Byrne EJ, Camus V, Caputo M, Collins D, De Deyn PP, Elina K, Frisoni G, Girtler N, Holmes C, Hurt C, Marriott A, Mecocci P, Nobili F, Ousset PJ, Reynish E, Salmon E, Tsolaki M, Vellas B, Robert PH: Neuropsychiatric syndromes in dementia. Results from the European Alzheimer Disease Consortium. Part I. Dement Geriatr Cogn Disord 2007;24:457-463.

- 16 Chen ST, Sultzer DL, Hinkin CH, Mahler ME, Cummings JL: Executive dysfunction in Alzheimer's disease: association with neuropsychiatric symptoms and functional impairment. J Neuropsychiatry Clin Neurosci 1998;10:426-432.
17 McPherson S, Fairbanks L, Tiken S, Cummings JL, Back-Madruga C: Apathy and executive function in Alzheimer's disease. J Int Neuropsychol Soc 2002;8:373-381.

18 Boyle PA, Malloy PF, Salloway S, Cahn-Weiner DA, Cohen R, Cummings JL: Executive dysfunction and apathy predict functional impairment in Alzheimer disease. Am J Geriatr Psychiatry 2003;11:214-221.

19 Marshall GA, Rentz DM, Frey MT, Locascio JJ, Johnson KA, Sperling RA: Alzheimer's Disease Neuroimaging Initiative: Executive function and instrumental activities of daily living in mild cognitive impairment and Alzheimer's disease. Alzheimers Dement 2011; 7:300-308.

-20 Okura T, Plassman BL, Steffens DC, Llewellyn DJ, Potter GG, Langa KM: Prevalence of neuropsychiatric symptoms and their association with functional limitations in older adults in the United States: the aging, demographics, and memory study. J Am Geriatr Soc 2010;58:330-337.

21 Palmer K, Lupo F, Perri R, Salamone G, Fadda L, Caltagirone C, Musicco M, Cravello L: Predicting disease progression in Alzheimer's disease: the role of neuropsychiatric syndromes on functional and cognitive decline. J Alzheimers Dis 2011;24:35-45.

-22 Scarmeas N, Brandt J, Blacker D, Albert M, Hadjigeorgiou G, Dubois B, Devanand D, Honig L, Stern Y: Disruptive behavior as a predictor in Alzheimer disease. Arch Neurol 2007;64:1755-1761.

23 Scarmeas N, Brandt J, Albert M, Hadjigeorgiou G, Papadimitriou A, Dubois B, Sarazin M, Devanand D, Honig L, Marder K, Bell K, Wegesin D, Blacker D, Stern Y: Delusions and hallucinations are associated with worse outcome in Alzheimer disease. Arch Neurol 2005;62:1601-1608.

24 Mok WY, Chu LW, Chung CP, Chan NY, Hui SL: The relationship between non-cognitive symptoms and functional impairment in Alzheimer's disease. Int J Geriatr Psychiatry 2004;19:1040-1046.

25 Mueller SG, Weiner MW, Thal LJ, Petersen RC, Jack C, Jagust W, Trojanowski JQ, Toga AW, Beckett L: The Alzheimer's Disease Neuroimaging Initiative. Neuroimaging Clin $\mathrm{N}$ Am 2005;15:869-877.

26 Sheikh JI, Yesavage JA: Geriatric Depression Scale (GDS): recent evidence and development of a shorter version; in Brink TL (ed): Clinical Gerontology: A Guide to Assessment and Intervention. New York, Haworth, 1986, pp 165-173.

27 Rosen WG, Terry RD, Fuld PA, Katzman R, Peck A: Pathological verification of ischemic score in differentiation of dementias. Ann Neurol 1980;7:486-488.

28 Morris JC: The Clinical Dementia Rating (CDR): current version and scoring rules. Neurology 1993;43:2412-2414.
29 Folstein MF, Folstein SE, McHugh PR: 'Minimental state'. A practical method for grading the cognitive state of patients for the clinician. J Psychiatr Res 1975;12:189-198.

30 Wechsler D: WMS-R: Wechsler Memory Scale-Revised Manual. New York, The Psychological Corporation, Harcourt Brace Jovanovich, 1987.

31 Petersen RC: Mild cognitive impairment as a diagnostic entity. J Intern Med 2004;256: 183-194.

32 McKhann G, Drachman D, Folstein M, Katzman R, Price D, Stadlan EM: Clinical diagnosis of Alzheimer's disease: report of the NINCDS-ADRDA Work Group under the auspices of Department of Health and Human Services Task Force on Alzheimer's Disease. Neurology 1984;34:939-944.

33 Kaufer DI, Cummings JL, Ketchel P, Smith V, MacMillan A, Shelley T, Lopez OL, DeKosky ST: Validation of the NPI-Q, a brief clinical form of the Neuropsychiatric Inventory. J Neuropsychiatry Clin Neurosci 2000;12: 233-239.

34 Rey A: L'examen clinique en psychologie. Paris, Presses Universitaires de France, 1964.

35 Wechsler D: WAIS-R manual. New York, The Psychological Corporation, 1981.

-36 Nelson HE, O'Connell A: Dementia: the estimation of premorbid intelligence levels using the New Adult Reading Test. Cortex 1978;14: 234-244.

37 Locascio JJ, Atri A: An overview of longitudinal data analysis methods for neurological research. Dement Geriatr Cogn Dis Extra 2011;1:330-357.

38 Buccione I, Perri R, Carlesimo GA, Fadda L, Serra L, Scalmana S, Caltagirone C: Cognitive and behavioural predictors of progression rates in Alzheimer's disease. Eur J Neurol 2007;14:440-446.

39 Burns A, Jacoby R, Levy R: Psychiatric phenomena in Alzheimer's disease. II. Disorders of perception. Br J Psychiatry 1990;157: 76-81, 92-94.

-40 Harwood DG, Barker WW, Ownby RL, Duara R: Relationship of behavioral and psychological symptoms to cognitive impairment and functional status in Alzheimer's disease. Int J Geriatr Psychiatry 2000;15: 393-400.

41 Lopez OL, Smith G, Becker JT, Meltzer CC, DeKosky ST: The psychotic phenomenon in probable Alzheimer's disease: a positron emission tomography study. J Neuropsychiatry Clin Neurosci 2001;13:50-55.

42 Holroyd S, Shepherd ML, Downs JH 3rd: Occipital atrophy is associated with visual hallucinations in Alzheimer's disease. J Neuropsychiatry Clin Neurosci 2000;12:25-28.

-43 Ott BR, Noto RB, Fogel BS: Apathy and loss of insight in Alzheimer's disease: a SPECT imaging study. J Neuropsychiatry Clin Neurosci 1996;8:41-46. 
-44 Craig AH, Cummings JL, Fairbanks L, Itti L, Miller BL, Li J, Mena I: Cerebral blood flow correlates of apathy in Alzheimer disease. Arch Neurol 1996;53:1116-1120.

-45 Migneco O, Benoit M, Koulibaly PM, Dygai I, Bertogliati C, Desvignes P, Robert $\mathrm{PH}$, Malandain G, Bussiere F, Darcourt J: Perfusion brain SPECT and statistical parametric mapping analysis indicate that apathy is a cingulate syndrome: a study in Alzheimer's disease and nondemented patients. Neuroimage 2001;13:896-902.

-46 Benoit M, Clairet S, Koulibaly PM, Darcourt J, Robert PH: Brain perfusion correlates of the apathy inventory dimensions of Alzheimer's disease. Int J Geriatr Psychiatry 2004;19: 864-869.

-47 Marshall GA, Monserratt L, Harwood D, Mandelkern M, Cummings JL, Sultzer DL: Positron emission tomography metabolic correlates of apathy in Alzheimer disease. Arch Neurol 2007;64:1015-1020.

48 Apostolova LG, Akopyan GG, Partiali N, Steiner CA, Dutton RA, Hayashi KM, Dinov ID, Toga AW, Cummings JL, Thompson PM: Structural correlates of apathy in Alzheimer's disease. Dement Geriatr Cogn Disord 2007; 24:91-97.
49 Marshall GA, Fairbanks LA, Tekin S, Vinters HV, Cummings JL: Neuropathologic correlates of apathy in Alzheimer's disease. Dement Geriatr Cogn Disord 2006;21:144-147.

50 Skogseth R, Mulugeta E, Jones E, Ballard C, Rongve A, Nore S, Alves G, Aarsland D: Neuropsychiatric correlates of cerebrospinal fluid biomarkers in Alzheimer's disease. Dement Geriatr Cogn Disord 2008;25: 559-563.

51 Petersen RC, Smith GE, Ivnik RJ, Tangalos EG, Schaid DJ, Thibodeau SN, Kokmen E, Waring SC, Kurland LT: Apolipoprotein E status as a predictor of the development of Alzheimer's disease in memory-impaired individuals. JAMA 1995;273:1274-1278.

52 Petersen RC, Thomas RG, Grundman M, Bennett D, Doody R, Ferris S, Galasko D, Jin S, Kaye J, Levey A, Pfeiffer E, Sano M, van Dyck CH, Thal LJ; Alzheimer's Disease Cooperative Study Group: Vitamin E and donepezil for the treatment of mild cognitive impairment. N Engl J Med 2005;352: 2379-2388.

53 Ritchie K, Artero S, Touchon J: Classification criteria for mild cognitive impairment: a population-based validation study. Neurology 2001;56:37-42.
54 Chen P, Ratcliff G, Belle SH, Cauley JA, DeKosky ST, Ganguli M: Cognitive tests that best discriminate between presymptomatic $\mathrm{AD}$ and those who remain nondemented. Neurology 2000;55:1847-1853.

55 Geda YE, Knopman DS, Mrazek DA, Jicha GA, Smith GE, Negash S, Boeve BF, Ivnik RJ, Petersen RC, Pankratz VS, Rocca WA: Depression, apolipoprotein E genotype, and the incidence of mild cognitive impairment: a prospective cohort study. Arch Neurol 2006; 63:435-440.

56 Rozzini L, Chilovi BV, Trabucchi M, Padovani A: Depression is unrelated to conversion to dementia in patients with mild cognitive impairment. Arch Neurol 2005;62: 505

57 Modrego PJ, Ferrandez J: Depression in patients with mild cognitive impairment increases the risk of developing dementia of Alzheimer type: a prospective cohort study. Arch Neurol 2004;61:1290-1293.

58 Spalletta G, Musicco M, Padovani A, Rozzini L, Perri R, Fadda L, Canonico V, Trequattrini A, Pettenati C, Caltagirone C, Palmer K: Neuropsychiatric symptoms and syndromes in a large cohort of newly diagnosed, untreated patients with Alzheimer disease. Am J Geriatr Psychiatry 2010;18: 1026-1035. 